\title{
An experimental study of the parallel and oblique vortex shedding from circular cylinders
}

\author{
By M. HAMMACHE AND M. GHARIB \\ Department of Applied Mechanics and Engineering Sciences, University of California, \\ San Diego, La Jolla, CA 92093, USA
}

(Received 7 February 1991 and in revised form 3 May 1991)

An experimental study of the origin of oblique vortex shedding in the laminar wake of circular cylinders was conducted in the range of Reynolds numbers from 40 to 160 . Two transverse circular cylinders were positioned upstream of the main shedding cylinder to control the angle of shedding from the main cylinder. The respective distances between each transverse cylinder and the main cylinder were used to induce oblique shedding of different angles, curved shedding, as well as parallel shedding. Measurements of the mean static pressure distribution in the base region of the cylinder and of the mean spanwise component of the velocity in the wake were taken. These measurements revealed that a non-symmetric pressure distribution, which induced a spanwise flow in the base region of the cylinder, was responsible for the oblique shedding. By using a simple model based on the ratio of the streamwise to the spanwise vorticity components, the angle of shedding was predicted within $2^{\circ}$ of the value measured from flow visualization. The vorticity was simply evaluated from the spanwise and streamwise velocity profiles of oblique vortex streets obtained with the LDV measurement technique. Parallel vortex shedding showed a symmetric pressure distribution with zero spanwise component of the velocity and zero crossshear in the cylinder base. It was shown that parallel vortex shedding results in a continuous Strouhal-Reynolds number curve.

\section{Introduction}

\subsection{Background and objective}

Past experiments on wakes of circular cylinders in a laminar flow have shown that the vortex shedding is two-dimensional only at low Reynolds numbers. As a certain Reynolds number, denoted here by $R e_{\mathrm{s}}$, is reached the vortex shedding becomes oblique, where the vortices are parallel to each other but inclined with respect to the cylinder axis. However, there is no consensus among various investigators on the value of the Reynolds number $R e_{\mathrm{s}}$ which marks the transition from parallel to oblique shedding.

An empirical relation which links the frequency of shedding from a circular cylinder to the free-stream velocity was proposed by Roshko (1954) as

$$
S t=0.212-4.5 / R e, \quad 50<R e<150,
$$

where $R e=U_{0} d / v$ is the Reynolds number (based on a cylinder of diameter $d$ in a free stream $U_{0}$ ), and $S t=f d / U_{0}$ is the Strouhal number (based on the vortex shedding frequency $f$ ). While using this relation to infer free-stream velocities from the frequency of shedding, Tritton (1959) found a discontinuity in the velocity-frequency curve. This discontinuity occurred at $R e \approx 90$ in wind tunnel experiments and 
$R e \approx 70$ in water channel experiments. Tritton did not link the appearance of the discontinuity to possible changes of the geometry of the shed vortices, but interpreted it instead as a transition in the nature of the flow instability from a vortex street originating in the wake to a vortex street originating in the immediate vicinity of the cylinder. Tritton's interpretation was disputed by several authors and various alternative scenarios were proposed. Gaster (1969) showed that frequency discontinuities could be caused by a slight shear in the approaching flow, or a slight taper of the cylinder. Similar conclusions were reached by Maull \& Young (1973) in their study of the wakes of circular cylinders in a shear flow. Slaouti \& Gerrard (1981) reported that even in a good quality flow and with a smooth and straight cylinder, the shed vortices are influenced by the geometry at the cylinder ends. For aspect ratios over 2500 and highly uniform upstream flow conditions, Van Atta \& Gharib (1987) showed that all discontinuities in the Strouhal-Reynolds number curve can be attributed to the flow-induced vibration of the cylinder. In experiments where the aspect ratio is not large enough, even with good flow conditions, minimizing the wall boundary-layer effect is difficult, if not impossible. Therefore, in many previous cylinder wake investigations where the aspect ratio usually does not exceed 300 (commonly regarded as a high aspect ratio), the wall-condition interference might have affected the natural two-dimensional shedding. Even in clean flow conditions and with the absence of cylinder vibration, eliminating the influence of the boundary layers on the wake of a cylinder is necessary to achieve two-dimensional vortex shedding. In fact, for cylinders of aspect ratio below 300, Williamson (1988) showed that discontinuities in the Strouhal-Reynolds number curve can be caused by the condition of non-parallel vortex shedding and are removed when parallel shedding is obtained.

The most basic approach to eliminate the end effects has been to use thin flat plates placed just outside the boundary layers at the wind or water tunnel walls. In this regard, Stansby (1974) determined that using end plates of suitable design resulted in a considerable reduction of the static pressure in the cylinder back-stagnation region. The design of such plates was largely based on considerations of the steadiness and spanwise uniformity of the cylinder base pressure. A study of the effect of the geometry at the cylinder ends on the angle and frequency of vortex shedding was conducted by Ramberg (1983), and in a recent study Williamson (1988) showed that end plates suitably angled with respect to the incoming flow can be used to induce parallel vortex shedding. Another approach to controlling the wake and which consists of end plates combined with larger diameter cylinders inserted at the ends of the main cylinder was presented by Eisenlohr \& Eckelmann (1989).

The mechanism by which any control technique operates to eliminate the oblique vortex shedding remains to be explained. The so-called 'transition' Reynolds number $R e_{\mathrm{s}}$ which marks the change of shedding geometry from parallel to oblique is known to remain fixed for a given flow facility, but the reported values of this Reynolds number are considerably scattered since no two flow facilities are identical. In a given facility, as the Reynolds number is gradually increased past the onset of shedding, the vortex geometry switches from parallel to oblique at a specific value of $R e=R e_{\mathrm{s}}$. This switch and the concomitant frequency discontinuity is initiated by the flow conditions at the cylinder ends. The dependency of this process on the Reynolds number would become questionable if it were demonstrated that oblique shedding is inducible at any $R e<R e_{\mathrm{s}}$. As will be described later, oblique shedding was produced at values of $R e<R e_{\mathrm{s}}$. The notion of a Reynolds-number-dependent secondary mode of instability is therefore examined here. 
We undertook a series of studies in order to clearly identify the mechanism responsible for the oblique vortex shedding. We created stable, reproducible oblique and parallel vortex shedding for which accurate pressure and velocity measurements were obtained to elucidate the differences between these two flow conditions. We attempted to use the angled end-plates technique to create these shedding geometries, but it was our experience that a certain amount of iteration was needed to operate successfully at all free-stream velocities and cylinder aspect ratios. An intuitive approach to the problem led us to develop an alternative control technique as described in Hammache \& Gharib (1989). As outlined in the next section, this technique, which we call the 'transverse control cylinders technique' or 'TCCT' for short, was easy to use and required no iteration when the velocity was varied, even at higher aspect ratios.

Various shedding geometries were induced by using the TCCT and relevant flow quantities, such as the mean pressure on the cylinder body, the mean streamwise and spanwise velocity components in the wake and the shedding frequency were measured carefully for each shedding geometry.

\subsection{Apparatus}

The experiments were conducted in two recirculating flow facilities : a wind tunnel with test section dimensions of $60.96 \mathrm{~cm}$ by $60.96 \mathrm{~cm}$ and a water tunnel with a test section of $25.4 \mathrm{~cm}$ by $25.4 \mathrm{~cm}$. The technique used to control the wake behind a circular cylinder consists of positioning two circular cylinders of diameter $D$ (referred to as the 'control cylinders') upstream of and normal to the shedding cylinder of diameter $d$ (referred to as the 'main cylinder'). The experimental arrangement with the relevant parameters is shown in figure 1 . The distances $H, L_{1}$ and $L_{2}$ can be adjusted by independently moving the control cylinders. The ratio $A=H / d$ defines an effective aspect ratio of the main cylinder. The region of interest is the region of the main cylinder span between the control cylinders. The main cylinder consisted of a stainless steel hypodermic tube of high outside diameter consistency $\left(1.27 \times 10^{-3} \mathrm{~cm}\right.$ precision) and very smooth surface finish. The control cylinders were stainless steel drill rods. Precautions were taken to avoid any vibration of the main cylinder. Vibration which could be transmitted by the tunnel itself was avoided by attaching the main cylinder to a frame outside the test section with no contact with the wind tunnel. Flow-induced vibration was eliminated by keeping the natural frequency of the main cylinder from interacting with the shedding frequency. This was achieved by applying an adequate tension on the cylinder in order to 'tune' the natural frequency away from any frequencies which would cause lock-on. This measure was supplemented by the use of foam pieces at the cylinder ends to dampen it. The cylinder displacement was monitored by an infrared photon coupled interrupter modulus (GE H13A1) which helped ensure that the cylinder was not vibrating.

The plan view of the wake was visualized by using the smoke wire technique in air and the hydrogen bubble technique in water. Both techniques use a thin stainless steel wire positioned parallel to the main cylinder. In air, the wire is coated with oil then heated to produce smoke. In water, the wire is the cathode of a DC circuit, and a thin copper plate placed farther downstream is the anode. The water is electrolysed by a pulsed voltage and a sheet of hydrogen bubbles is generated at the wire and carried away by the flow. In both techniques, the wire was downstream of the cylinder and at one edge of the Kármán vortex street, so that only one side of the vortex street was traced. 


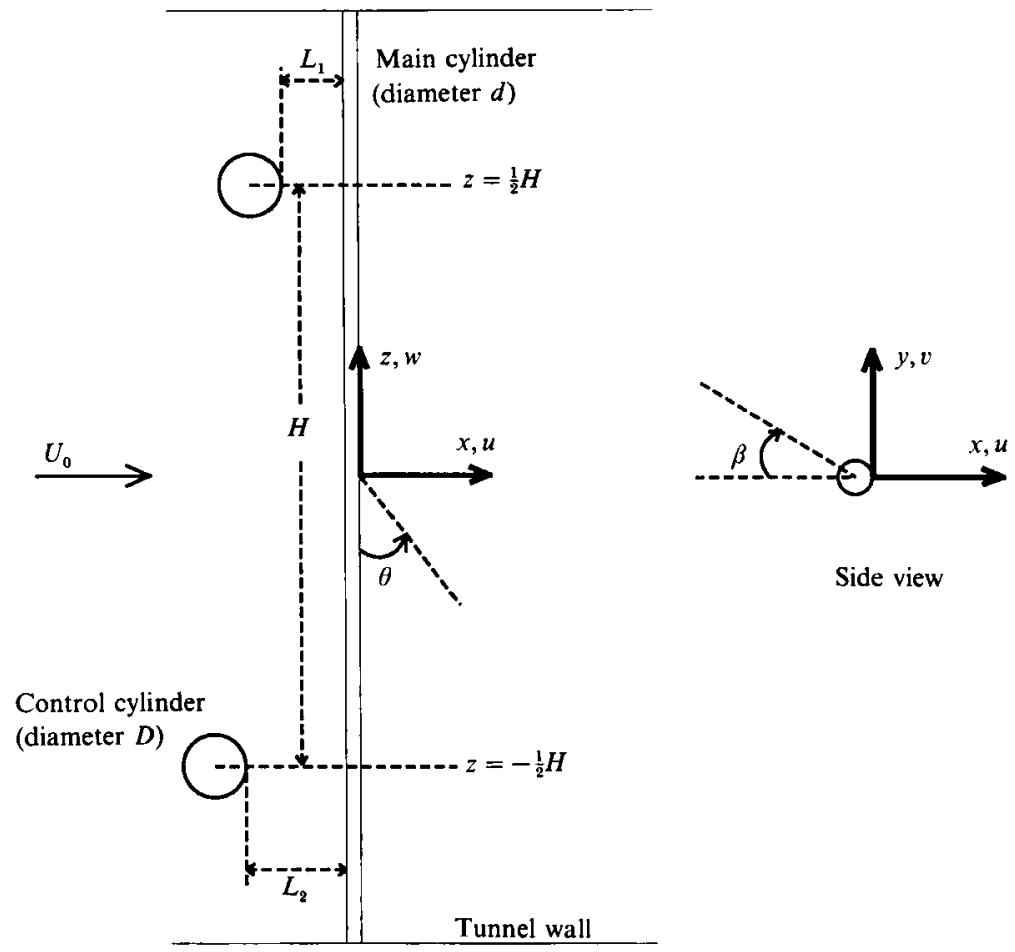

Plan view

FIGURE 1. Schematics of the experimental set-up and reference coordinate system.

Measurements of the mean static pressure on the surface of the main cylinder were taken in the wind tunnel experiments. A very small hole (typically of $2.5 \times 10^{-3} \mathrm{~cm}$ diameter) was tapped on the hypodermic tube by using an Electro-Discharge Machine, and one end of the tube was connected to the active port of an accurate differential pressure transducer (MKS, model 398) while the other end was sealed. The reference port of the pressure sensor was connected to the static tap of a United Sensor Pitot-static tube. The spanwise distribution of pressure was obtained by sliding the main cylinder along its axis, thus moving the location of the pressure tap along the cylinder span.

The frequency spectra were measured in the wind tunnel with a hot wire (TSI, model 1210-T1.5) fed into a two-channel FFT signal analyser (Scientific Atlanta, model SD380Z). Velocity data were taken in the water tunnel with a two-channel laser-Doppler velocimeter (Dantec fibre optics LDV). All data, in both wind and water tunnel experiments, were sampled with a 12-bit $A / D$ converter on a MASSCOMP computer.

The origin of the reference coordinate system was at the base of the cylinder, at the middle of the span; $x$ was measured in the streamwise direction, with $x=0$ at the back stagnation point of the cylinder, $y$ was measured in the direction normal to the free stream and normal to the cylinder axis, with $y=0$ at the centre of the wake, and $z$ was measured in the direction perpendicular to the plane $(x, y)$ with $z=0$ at the middle of the span of the cylinder.

When the control cylinders were in use, the coordinate $z$ was in the range $-\frac{1}{2} H$ to 


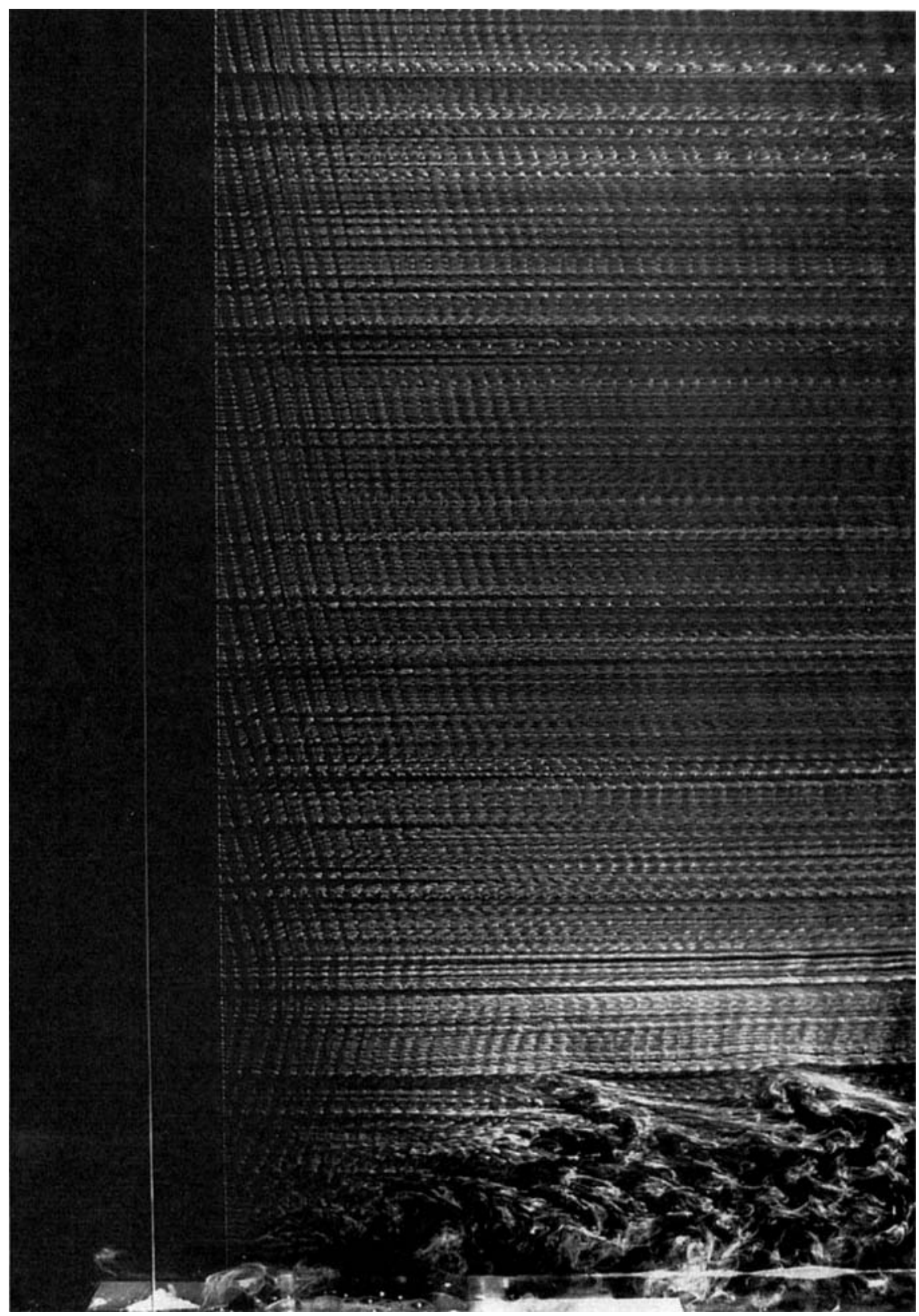

FraUre 2. Plan view of the wake in oblique shedding. $R e=100 ; d=0.08 \mathrm{~cm}$. Aspect ratio $=750$ based on test section width.

$+\frac{1}{2} H$, such that $A=H / d$ was the effective aspect ratio of the cylinder span. The angle of vortex shedding $\theta$ was measured counterclockwise from the $z$-axis. The angle $\beta$, measured from the front stagnation point of the main cylinder, represented the azimuthal coordinate on the cylinder surface. 


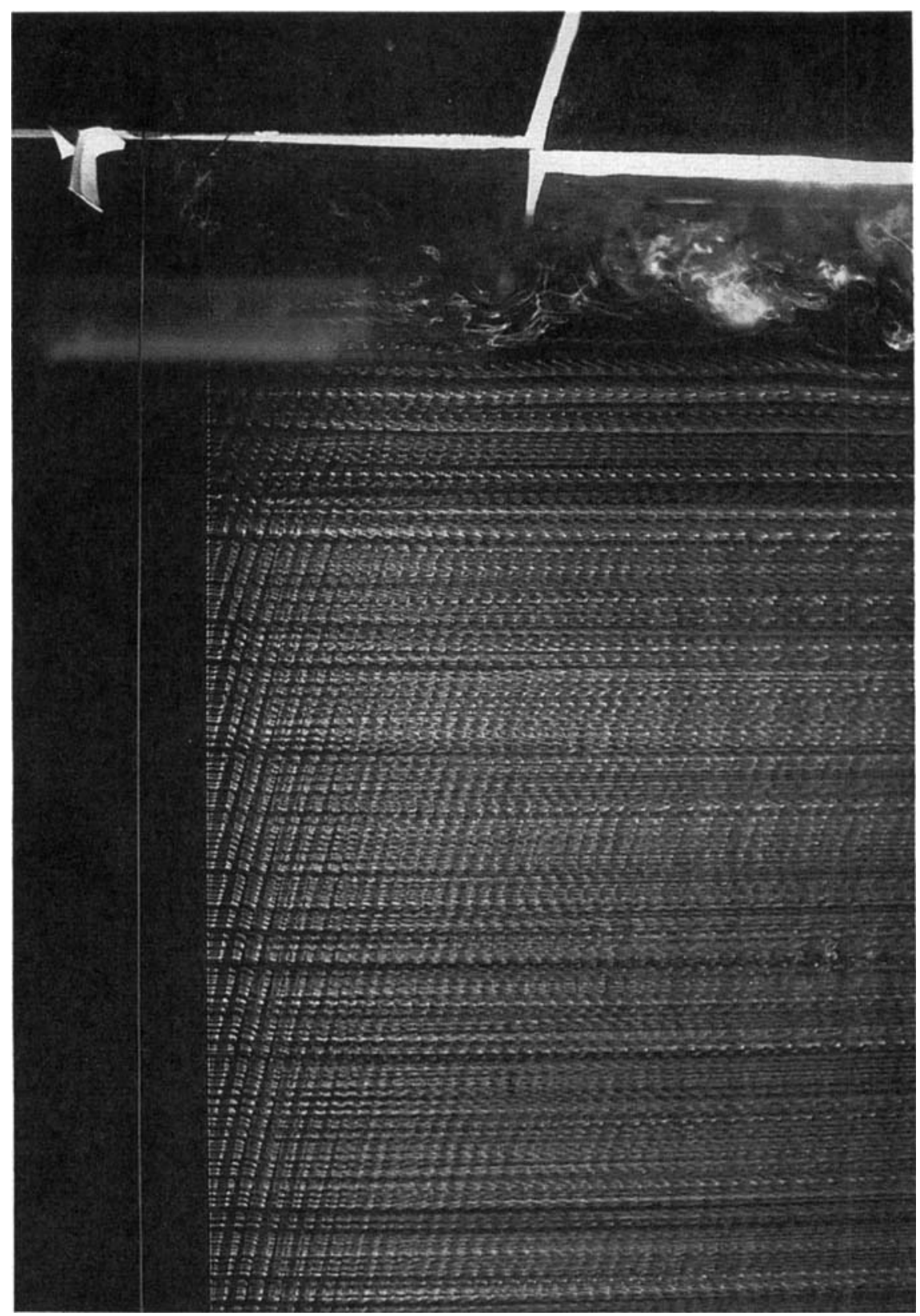

FIGURE 3. The effect of altering the wall boundary layer upstream of the upper end of the cylinder. $R e=100, d=0.08 \mathrm{~cm}$. Aspect ratio $=750$ based on test section width.

\section{Results and discussion}

2.1. The Kärmán vortex street: preliminary observations

In our wind tunnel facility, the oblique vortex shedding starts at $R e_{\mathrm{s}} \approx 72$ and dominates the wake until the three-dimensional transition to turbulence. Figure 2 presents the plan view of the wake in oblique shedding of about $13^{\circ}$ for a cylinder of aspect ratio 750 (based on the width of the test section), at $R e=100$. It is important 


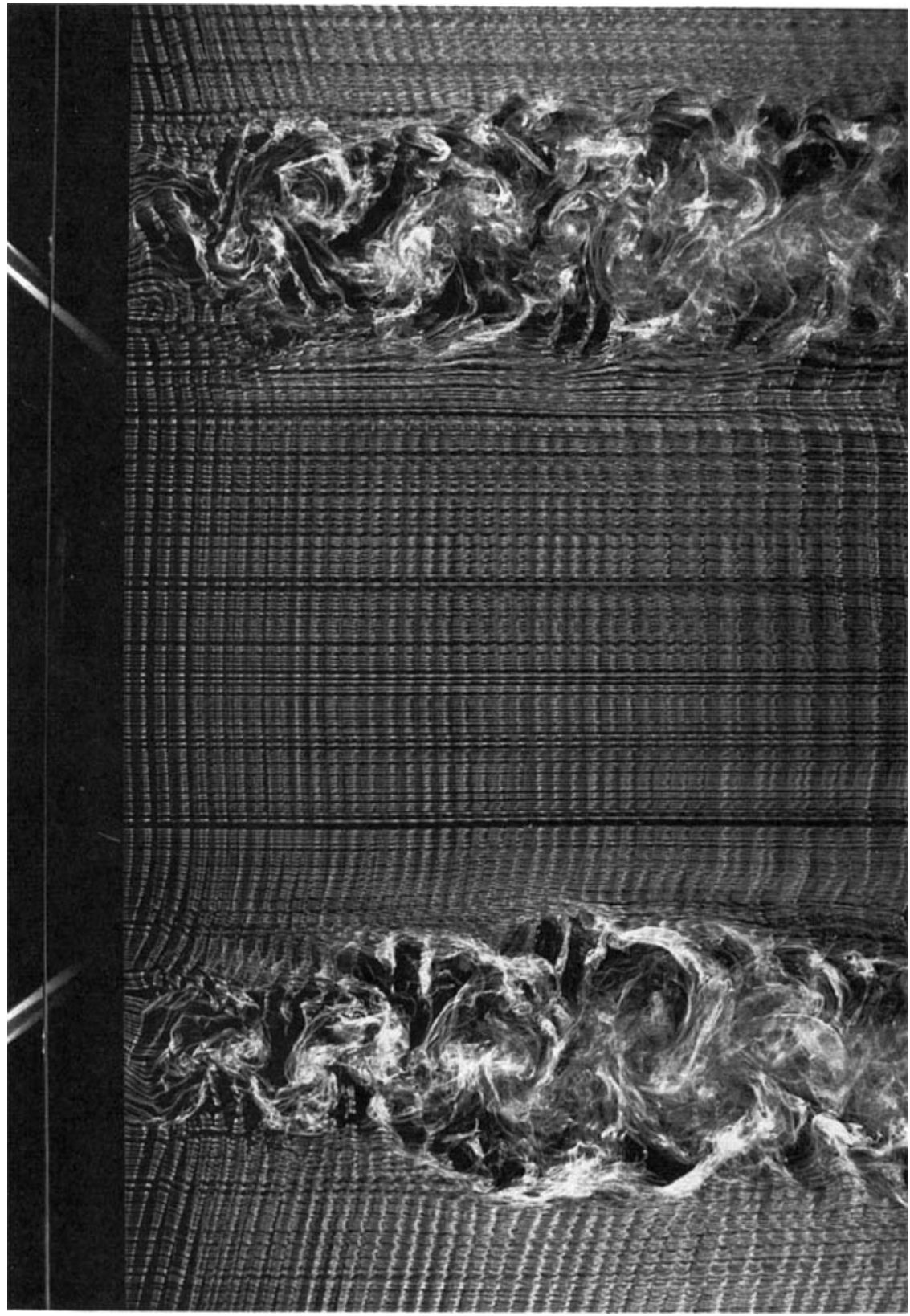

Figure 4. Parallel shedding obtained when the control cylinders are used. $R e=100$, $d=0.08 \mathrm{~cm}, D=0.78 \mathrm{~cm}, A=100, L=L_{0}$.

to note that even at this large aspect ratio the vortex shedding can be oblique. As we mentioned in §1.1, Van Atta \& Gharib had to use cylinders of aspect ratio above 2500 in a highly uniform flow and in the absence of cylinder vibration to obtain twodimensional laminar wakes.

In order to ensure that the oblique shedding is not due to flow non-uniformities, velocity profiles were taken across the test section of the wind tunnel, in the absence of any cylinders, at velocities which correspond to $R e=46,72$, and 111, based on the 
(a)

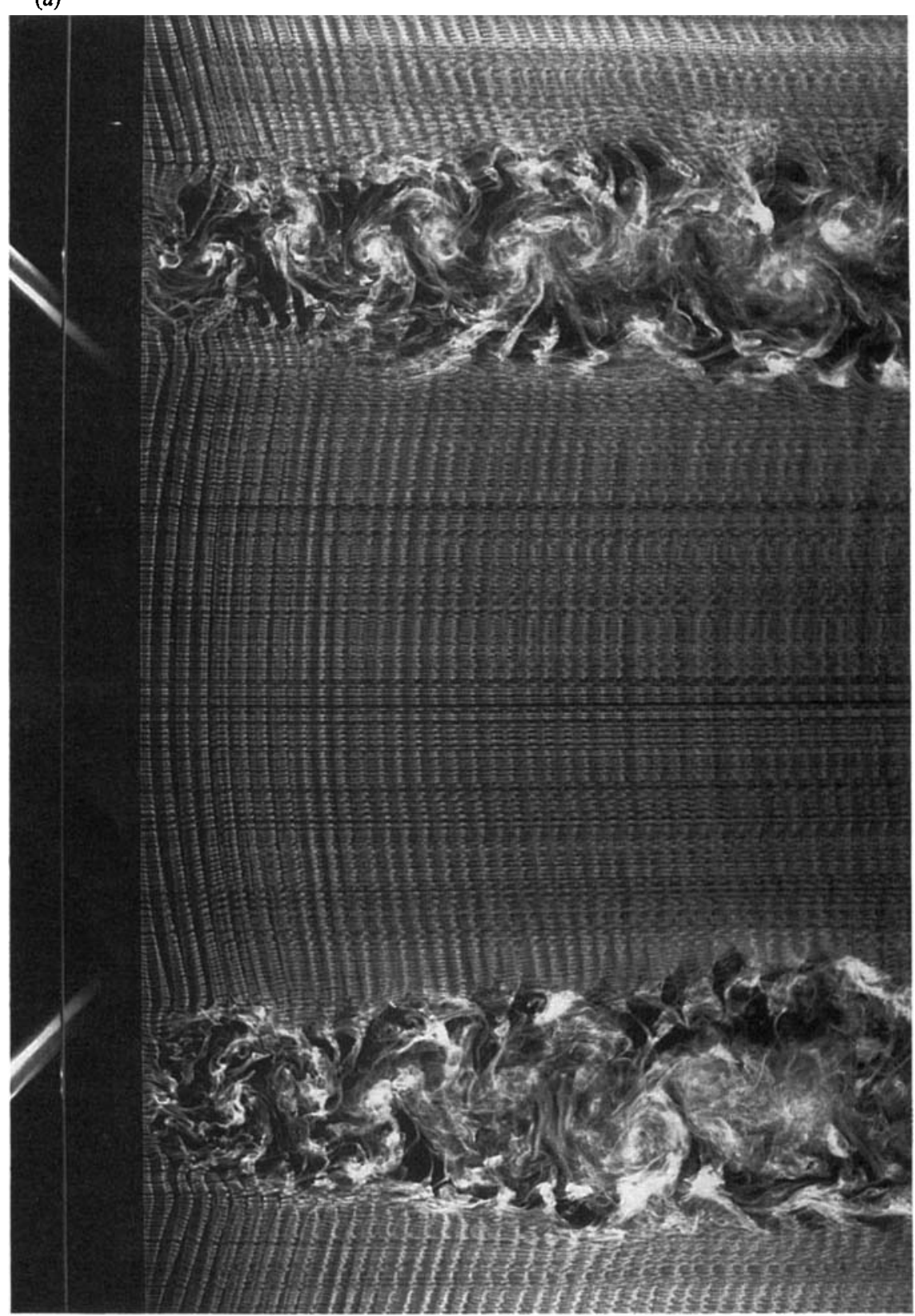

Figure 5(a). For caption see facing page.

diameter of our main cylinder. All three profiles showed a uniform flow across the test section. The free-stream velocity across $95 \%$ of the test section width was within $0.3 \%$ of the value at the centre of the test section.

The interaction between the wall boundary layer and the cylinder wake near the ends is so complex that no simple prediction could be made of the angle of vortex shedding. Evidently the flow conditions at the two ends are not identical, and it is their relative influences that control the angle of the vortices. As the flow velocity is 
(b)

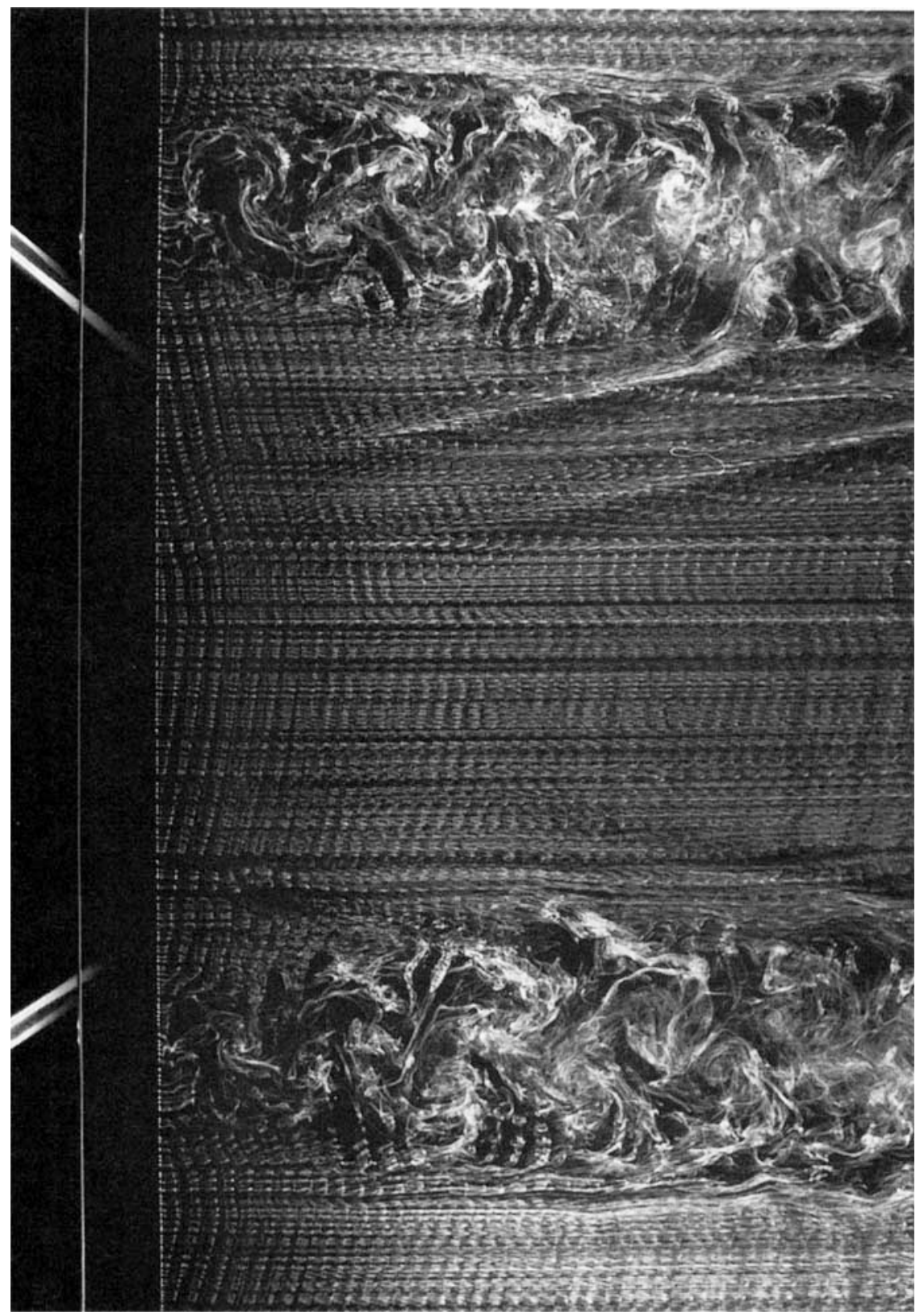

FIGURE 5. Curved vortex shedding obtained with the control cylinders. $R e=100 ; d=0.08 \mathrm{~cm}$, $D=0.78 \mathrm{~cm}, A=100$. (a) $L_{1}=L_{2}<L_{0}$, (b) $L_{1}=L_{2}>L_{0}$.

varied, the shedding angle $\theta$ changes in magnitude and/or sign, but the vortices adopt a stable geometry. Frequency jumps were observed at some velocities and the flow visualization showed an unstable configuration whereby the angle $\theta$ varied constantly, the wake being unable to find a stable geometry. The mechanism by which this unstable condition was initiated is not entirely clear. It may have been a result of changing relative strength of the horseshoe vortices at free-stream velocities 


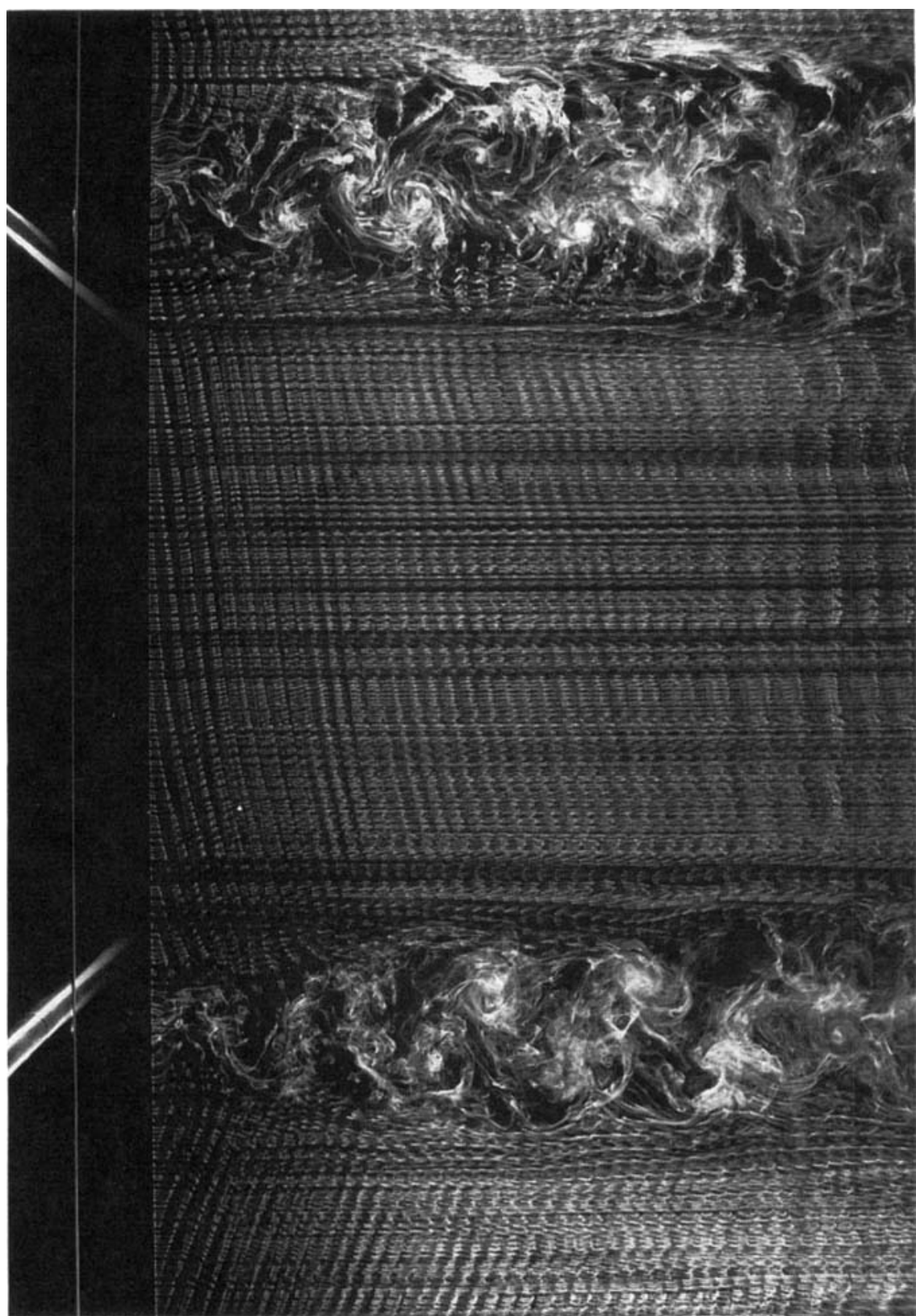

Frgure 6. Oblique shedding induced by the control cylinders at $R e=100, d=0.08 \mathrm{~cm}$, $D=0.78 \mathrm{~cm}, A=100$.

which produce unstable shedding near the ends. Note that the observed shedding angles and the location of the discontinuities on the $S t-R e$ curve should not be generalized for other flow facilities or other cylinder end geometries.

The sensitivity of the shedding angle to the flow conditions at the ends of the cylinder is obvious if one compares the angles of shedding in figures 2 and 3 . Figure 3 was obtained by positioning a thin square plate, of $1 \mathrm{~cm}$ width, normal to the wall and upstream of the upper end of the cylinder. The effect of this obstacle in the 


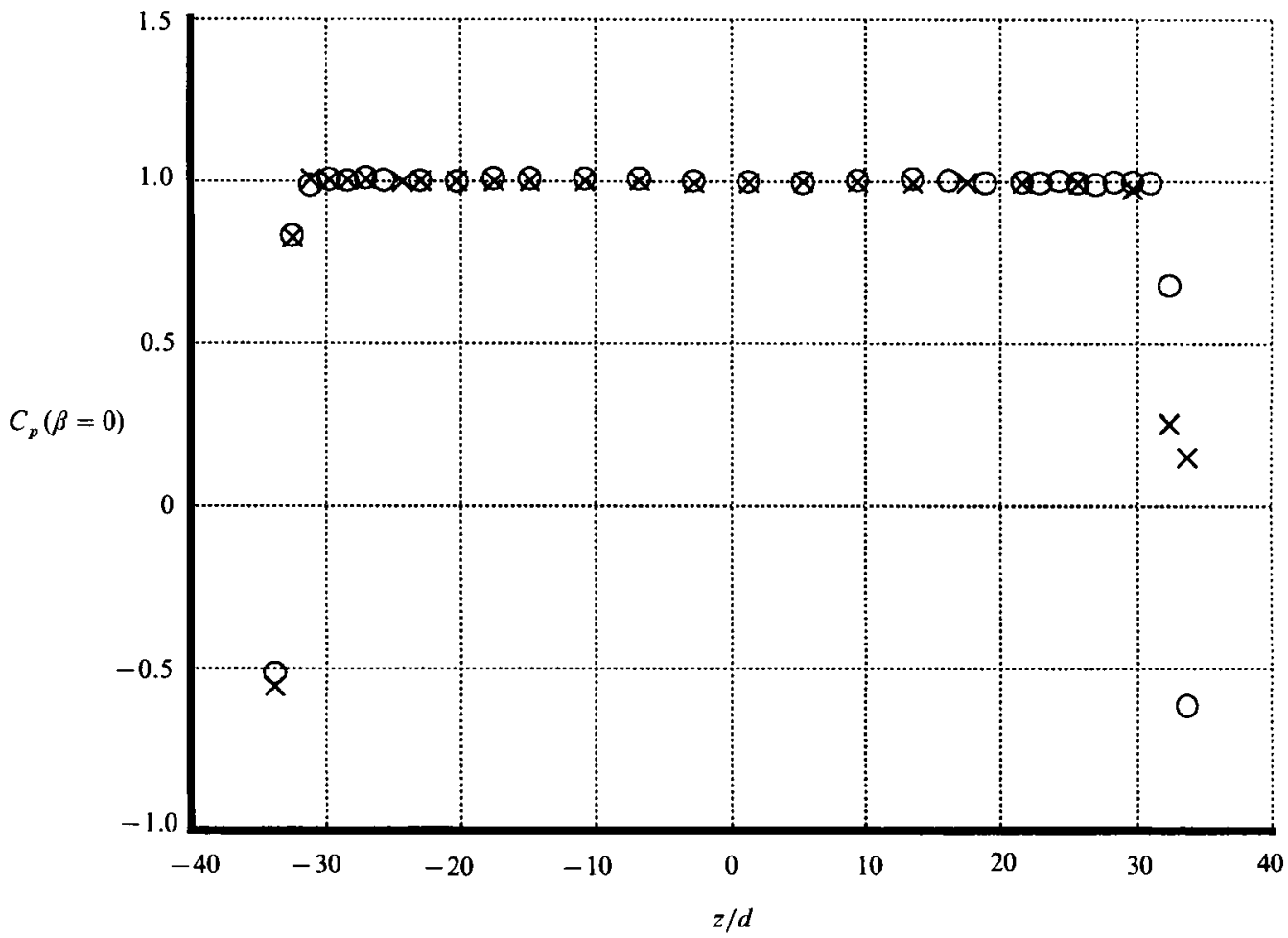

FIGURe 7. Spanwise distribution of the mean static pressure coefficient at the front stagnation point. $R e=121, d=0.08 \mathrm{~cm}, D=0.63 \mathrm{~cm}, A=75$. $\bigcirc$, Parallel shedding; $\times$, oblique shedding with $\theta<0$.

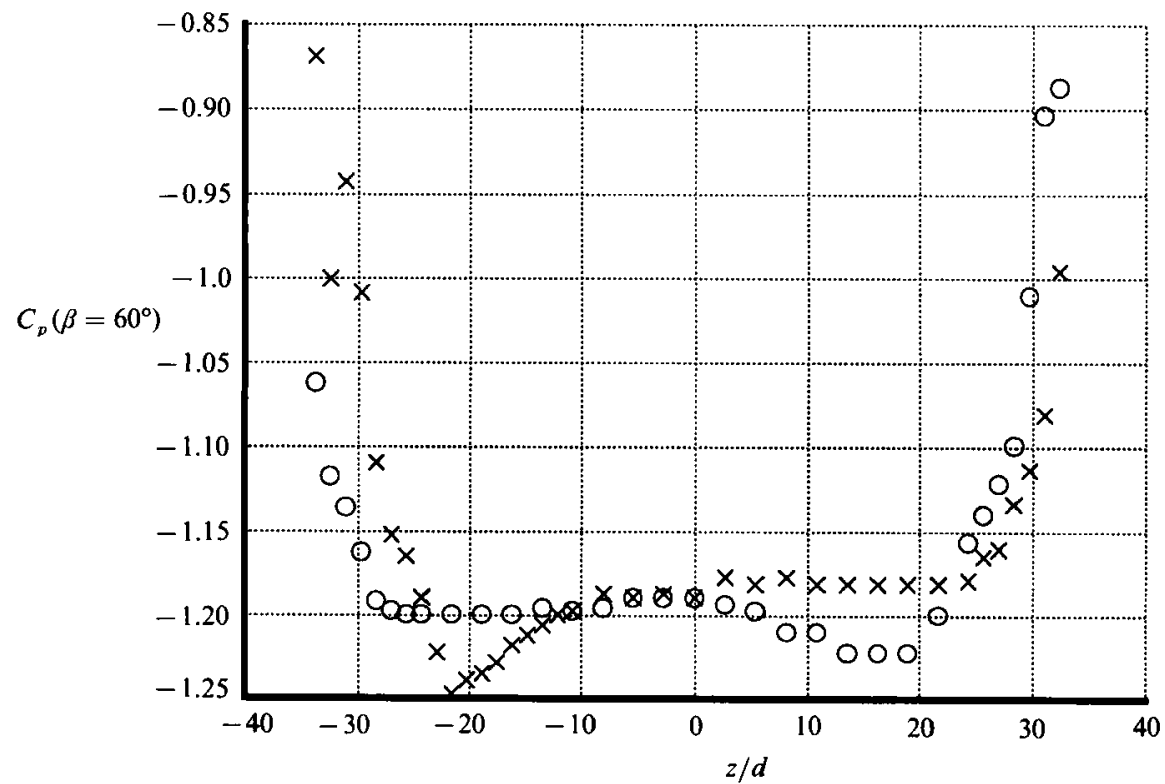

FIGURE 8. Spanwise distribution of mean static pressure coefficient at $\beta=60^{\circ}$. $R e=121$, $d=0.08 \mathrm{~cm}, D=0.63 \mathrm{~cm}, A=75$. $O$, Oblique shedding with $\theta<0 ; \times$, oblique shedding with $\theta>0$. 

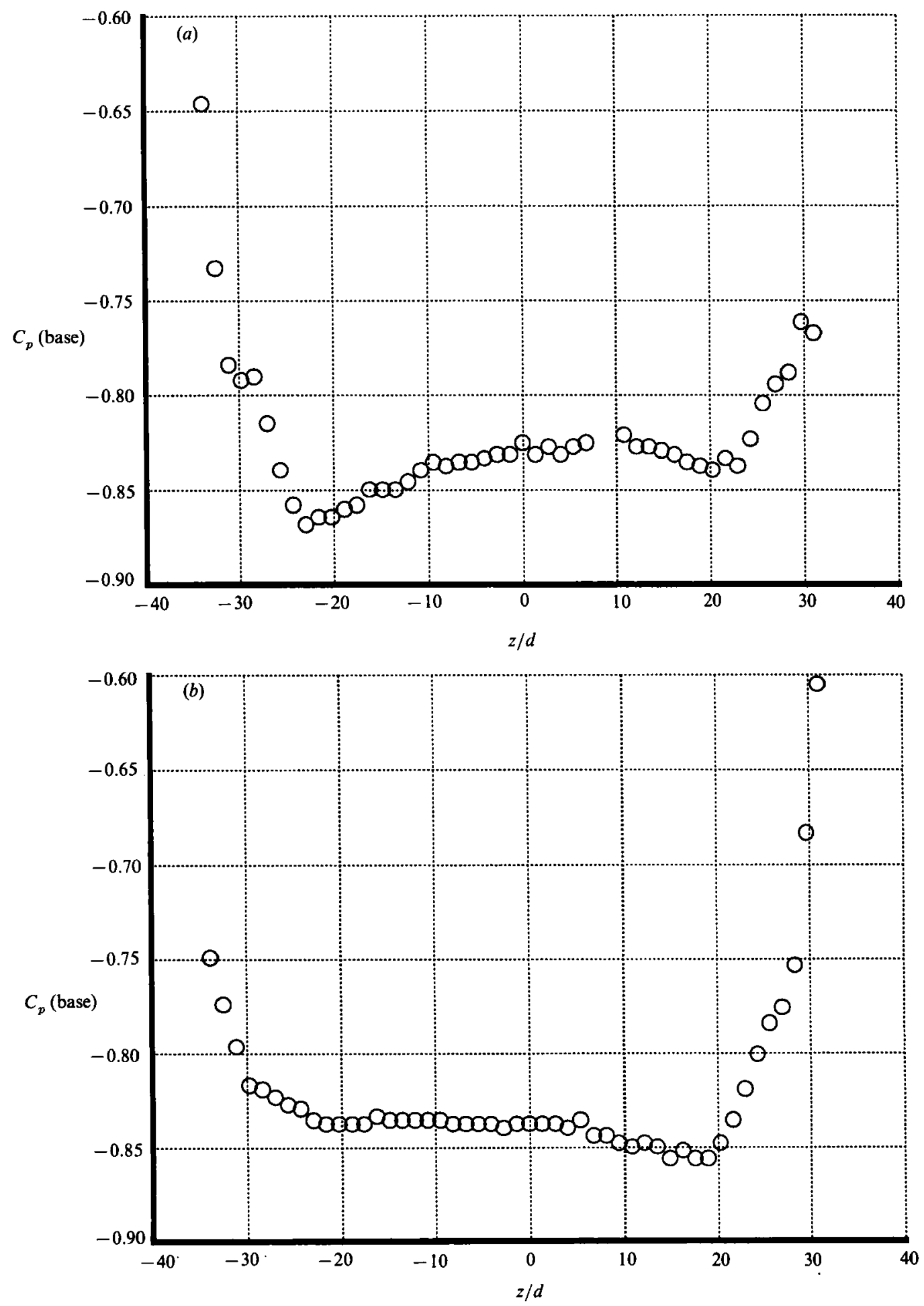

Figure $9(a, b)$. For caption see facing page. 


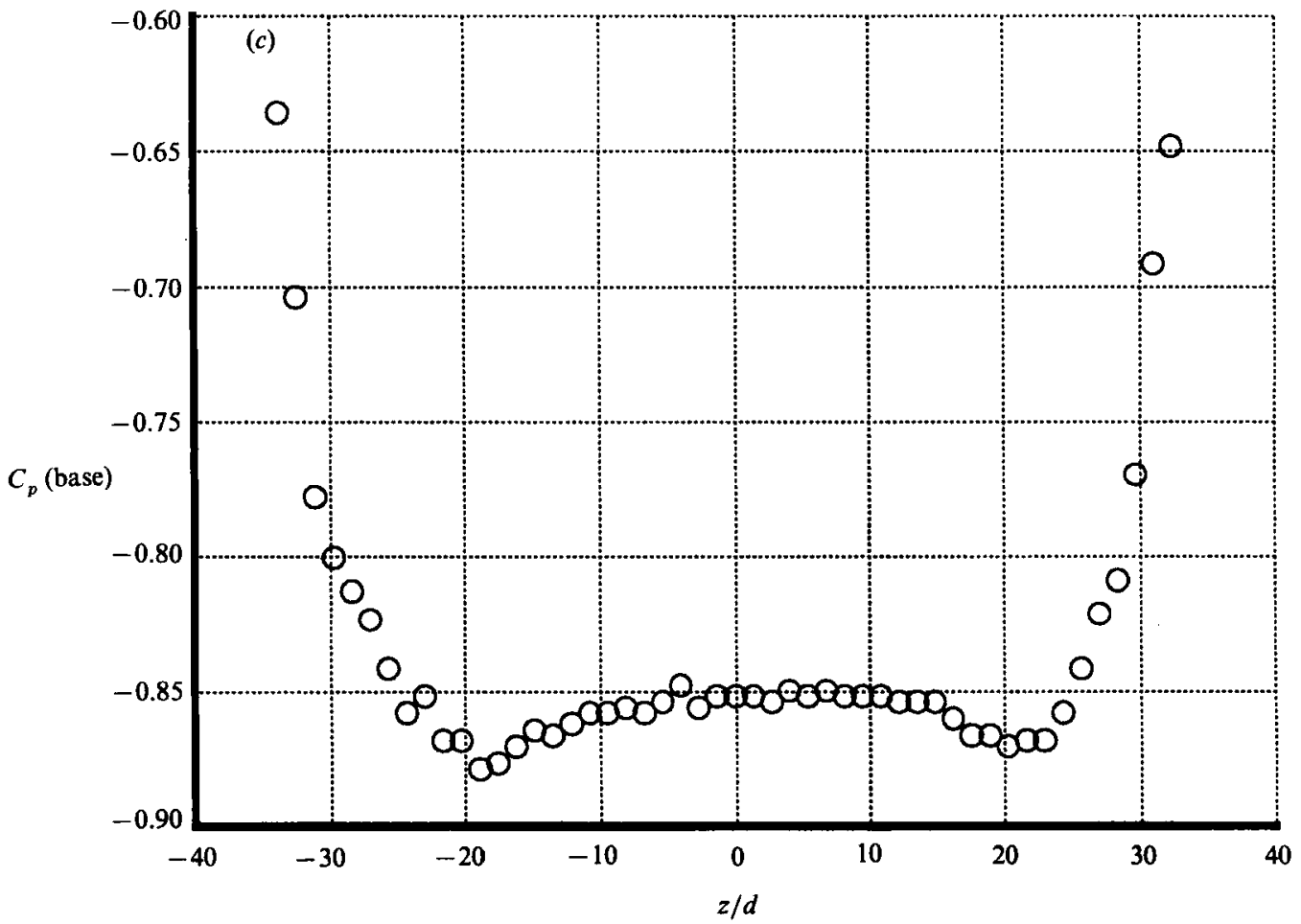

FIgURE 9. Spanwise distribution of mean base static pressure coefficient. $R e=121, d=0.08 \mathrm{~cm}$, $D=0.63 \mathrm{~cm}, A=75$. (a) Oblique shedding with $\theta>0$, (b) oblique shedding with $\theta<0,(c)$ parallel shedding.

boundary layer resulted in a change of both the sign and magnitude of the shedding angle, which changed from $13^{\circ}$ to $-15^{\circ}$. This is indicative of a direct dependence of the angle of vortex shedding upon the wall boundary-layer conditions at the ends of the cylinder. The scatter in the data found in the literature is inevitable because no two flow facilities are identical, at least as far as the wall boundary layers are concerned. The TCCT substitutes strict control of the cylinder wake for the arbitrary effect of the wall boundary layers. A detailed description of the various shedding geometries attained through the use of this technique is given below.

\subsection{The effect on the vortex geometry of controlling the cylinder ends}

Parallel vortex shedding is obtained by positioning the control cylinders at a specific distance $L_{1}=L_{2}=L_{0}$. This is illustrated in figure 4 at $R e=100$. The parameter $L_{0}$, for which the vortices in the controlled region are straight and parallel to the axis of the main cylinder, is a function only of the diameter $D$ of the control cylinders. Varying the free-stream velocity or the distance $H$ (which is equivalent to varying the effective aspect ratio $A=H / d$ ) has no effect on the shedding geometry. The vortices remain parallel to the cylinder axis in the controlled region.

For the symmetric arrangement $L_{1}=L_{2}=L$ but $L \neq L_{0}$, the vortex filaments are not straight. Instead, they are curved. Figure $5(a, b)$ shows the shape of the vortices for $L<L_{0}$ and $L>L_{0}$, respectively, at $R e=100$.

The more general case $L_{1} \neq L_{2}$ produces oblique shedding where the magnitude 


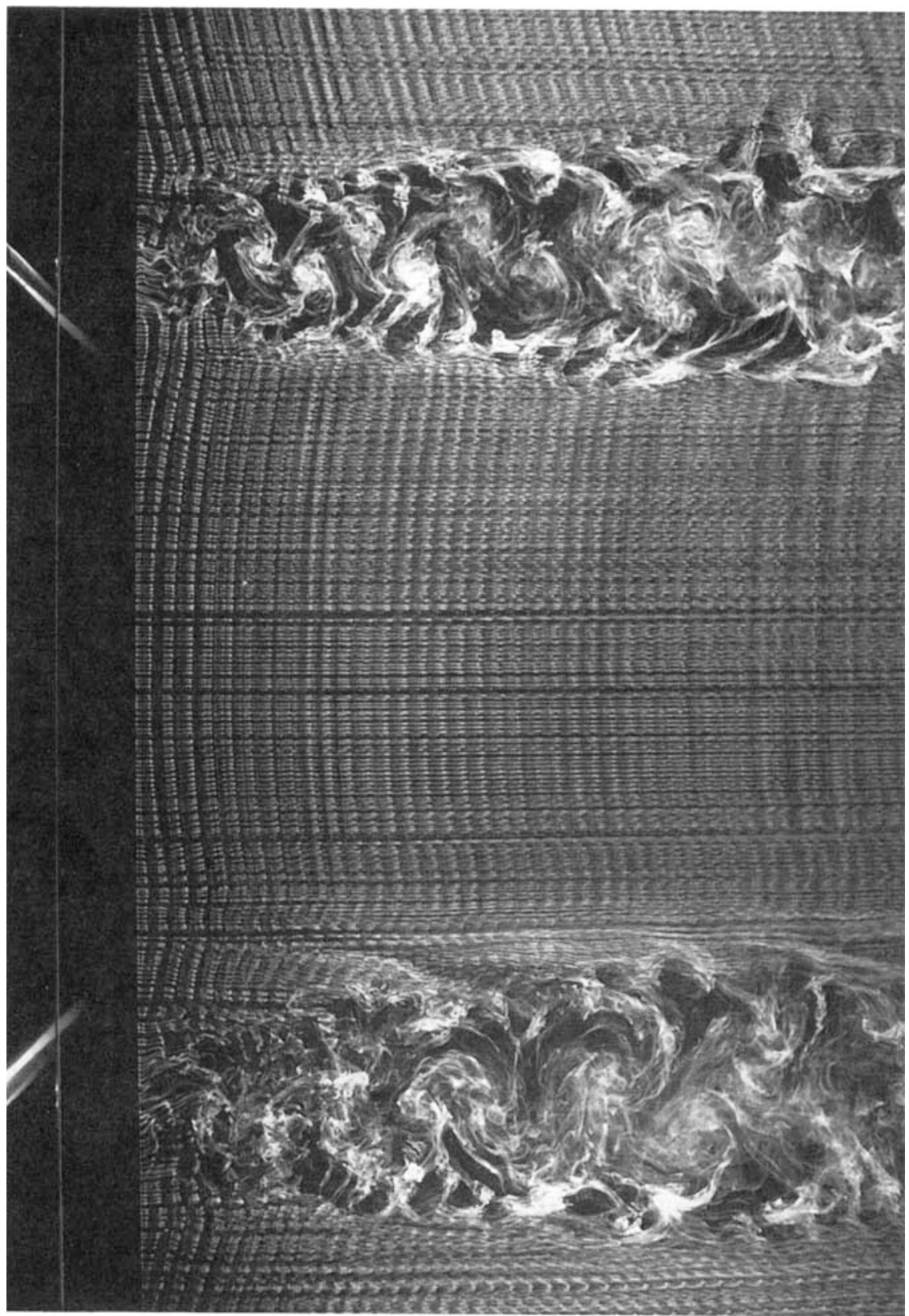

Figure 10. Parallel shedding obtained with two control cylinders of different diameters, $D_{1}=0.63 \mathrm{~cm}$ and $D_{2}=0.78 \mathrm{~cm} . R e=100, d=0.08 \mathrm{~cm}, A=100$.

and sign of the shedding angle depends on the specific values of $L_{1}$ and $L_{2}$. An example is shown in figure 6 .

Note that positioning the control cylinders downstream of the main cylinder or using only one control cylinder does not create parallel vortex shedding.

To understand the mechanism by which the TCCT (or any other control technique) works to induce parallel shedding one must study the flow conditions imposed on the cylinder ends and the extent of these conditions on the flow along the whole span of 


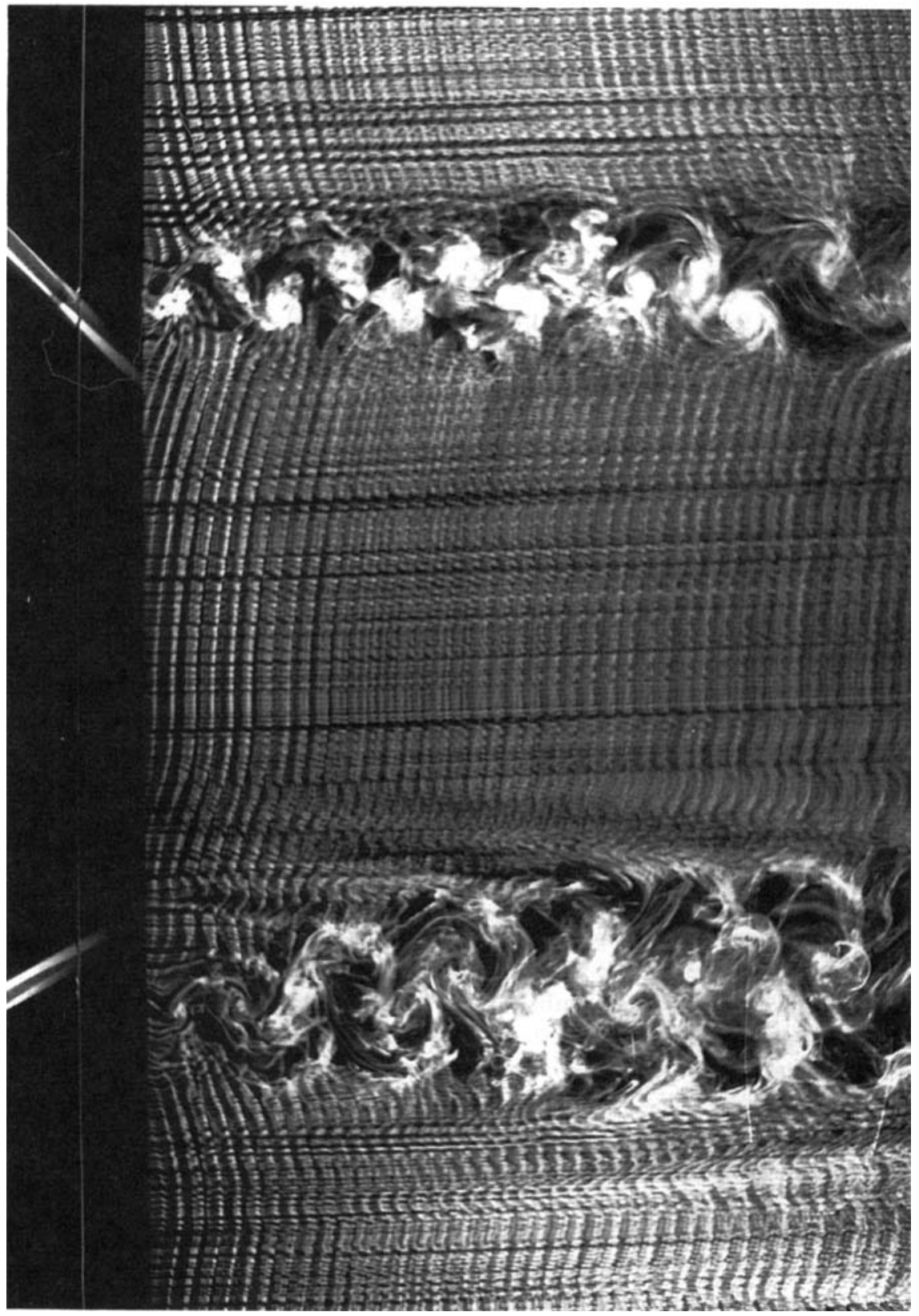

Figure 11. Oblique shedding obtained with two control cylinders of the same diameter, $D=0.78 \mathrm{~cm}$, but staggered. $R e=65, d=0.08 \mathrm{~cm}, A=100$.

the cylinder. We investigated the pressure and velocity fields for the different shedding geometries and the results are presented below.

\subsection{Static pressure measurements for parallel and oblique shedding}

As conjectured by Ramberg (1983), the base pressure near the ends of the cylinder can be influenced by end plates which are angled with respect to the free stream. Williamson (1988) attributed the success of the angled-end-plates technique to the 


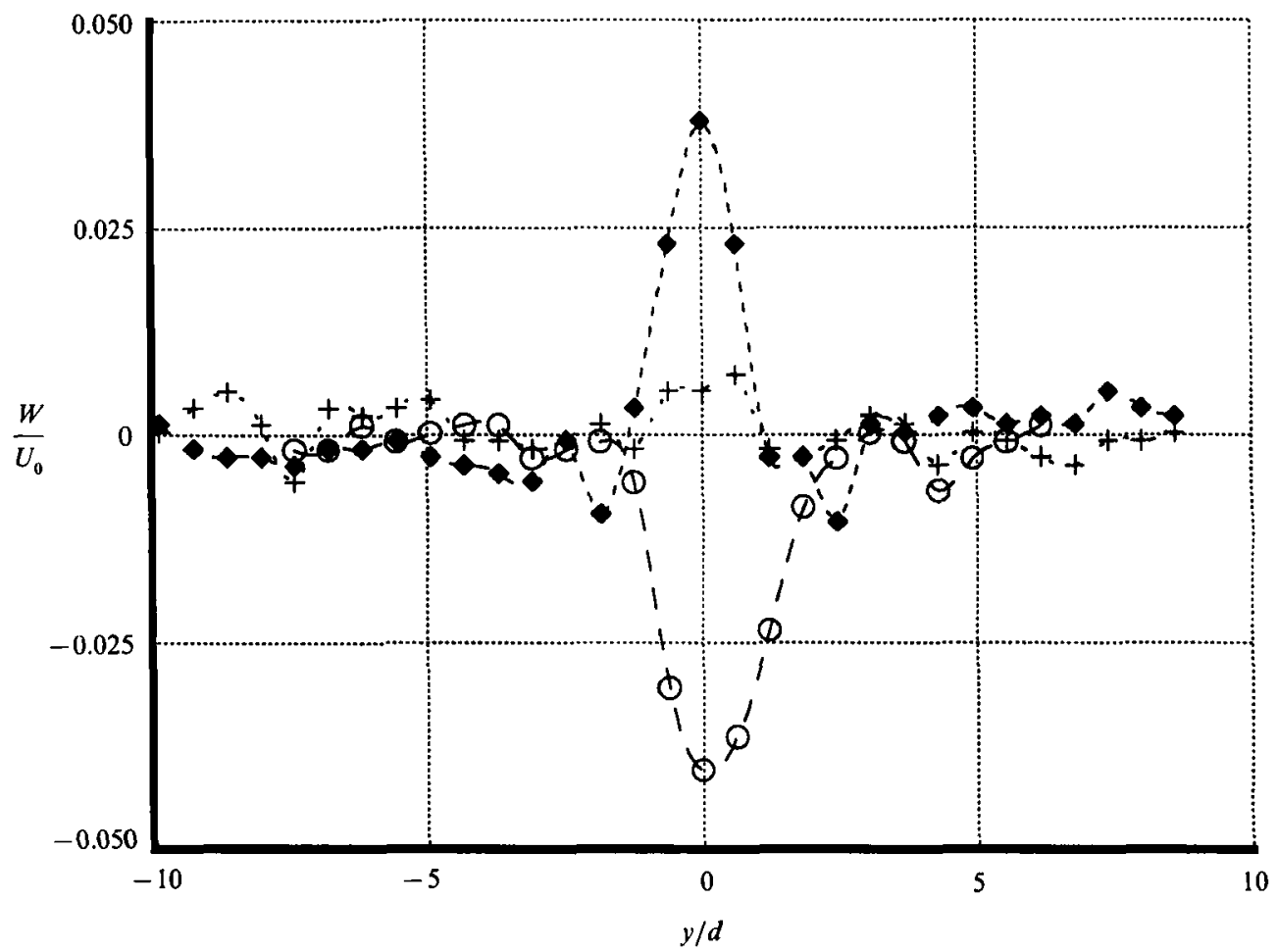

Figure 12. Profiles of the mean spanwise component of velocity at centre-span. $x / d=1$, $d=0.10 \mathrm{~cm}, D=0.47 \mathrm{~cm}, R e=100, A=190$. $O$, Oblique shedding with $\theta>0 ; \diamond$, oblique shedding with $\theta<0$; + parallel shedding.

stretching of the vortices by the trailing edge of the end plates, and later (Williamson 1989) considered the base pressure at the cylinder ends to be the cause of oblique vortex shedding. However, in a subsequent publication Williamson \& Roshko (1989) presented base pressure data over half the span of the cylinder where a substantial base pressure variation was not observed. In a model proposed by Hammache \& Gharib (1989), a spanwise pressure difference is also considered as a necessary condition for the existence of oblique shedding. To support this model, we present data on the pressure distribution in the base of the cylinder for the parallel and oblique shedding cases.

Measurements were taken of the mean static pressure along the span of the main cylinder, from $z=-\frac{1}{2} H$ to $z=\frac{1}{2} H$, at $\beta=0,60^{\circ}$ and $180^{\circ}$. These measurements were taken for the following vortex shedding geometries:

(i) parallel vortex shedding with $L_{1}=L_{2}=L_{0}$, i.e. $\theta=0$;

(ii) oblique vortex shedding with $L_{1}=L_{0}$ and $L_{2}>L_{0}$, i.e. $\theta<0$;

(iii) oblique vortex shedding with $L_{1}>L_{0}$ and $L_{2}=L_{0}$, i.e. $\theta>0$.

As seen in figure 7 , the front stagnation pressure distributions are identical, which suggests that the upstream flow conditions are not responsible for the oblique vortex shedding.

However, the base region is characterized by a symmetric pressure distribution when parallel vortex shedding is achieved. This symmetry is lost in the case of oblique shedding. This trend is very apparent in figures 8 and 9 , for $\beta=60^{\circ}$ and $\beta=180^{\circ}$ respectively. 


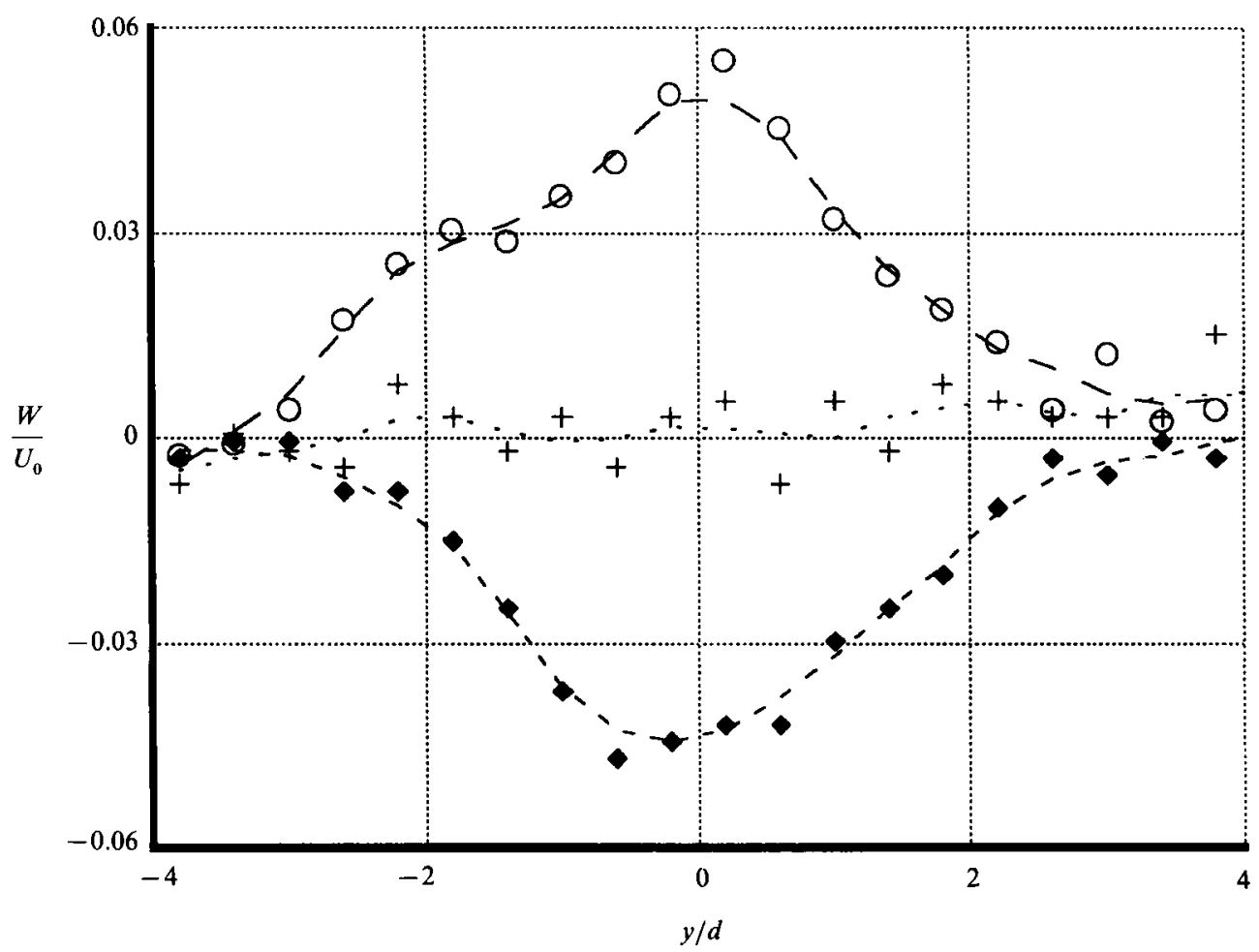

Figure 13. Profiles of the mean spanwise component of velocity for the curved shedding shown in figure $5(a) . R e=100, x / d=10, d=0.10 \mathrm{~cm}, D=0.47 \mathrm{~cm}, A=190.0, z / d=45 ;+, z / d=0 ;$ $z / d=-\mathbf{4 5}$.

The pressure measurements also help explain how the control cylinders can induce different vortex shedding geometries. The parameters $L_{1}$ and $L_{2}$ simply control the static pressure induced by the control cylinders on the main cylinder in the regions around $z=-\frac{1}{2} H$ and $z=\frac{1}{2} H$. When the imposed static pressures are equal, the vortex shedding is symmetrical. This, however, is not a sufficient condition for parallel shedding. In general, $L_{1}=L_{2}=L$ produces curved vortices, such as the cases shown in figure 5. To achieve parallel shedding, the imposed pressure distribution has to be uniform over the centre span. For $L<L_{0}$, the pressure in the central region is higher than at the ends, and conversely for $L>L_{0}$.

We conducted three experiments which support the above argument. Based on our model, one should be able to obtain a symmetric pressure boundary condition by using two control cylinders of different diameters. Figure 10 shows that parallel shedding could be induced by two control cylinders of diameters $D_{1}$, positioned at $L_{1}$, and $D_{2}$, positioned at $L_{2}$. Note that $L_{1} \neq L_{2}$ because each control cylinder had to be positioned at its optimum distance upstream of the main cylinder to create parallel shedding.

The second experiment was conducted at $R e=65$. This corresponds to naturally parallel shedding, since this Reynolds number is below the onset of the oblique shedding in our tunnel. By using control cylinders of the same size but such that $L_{1} \neq L_{2}$, the region of the wake between the control cylinders could be made oblique, whereas the outside regions remained parallel. This case is depicted in figure 11 .

In the third experiment, we placed the control cylinders downstream of the main 

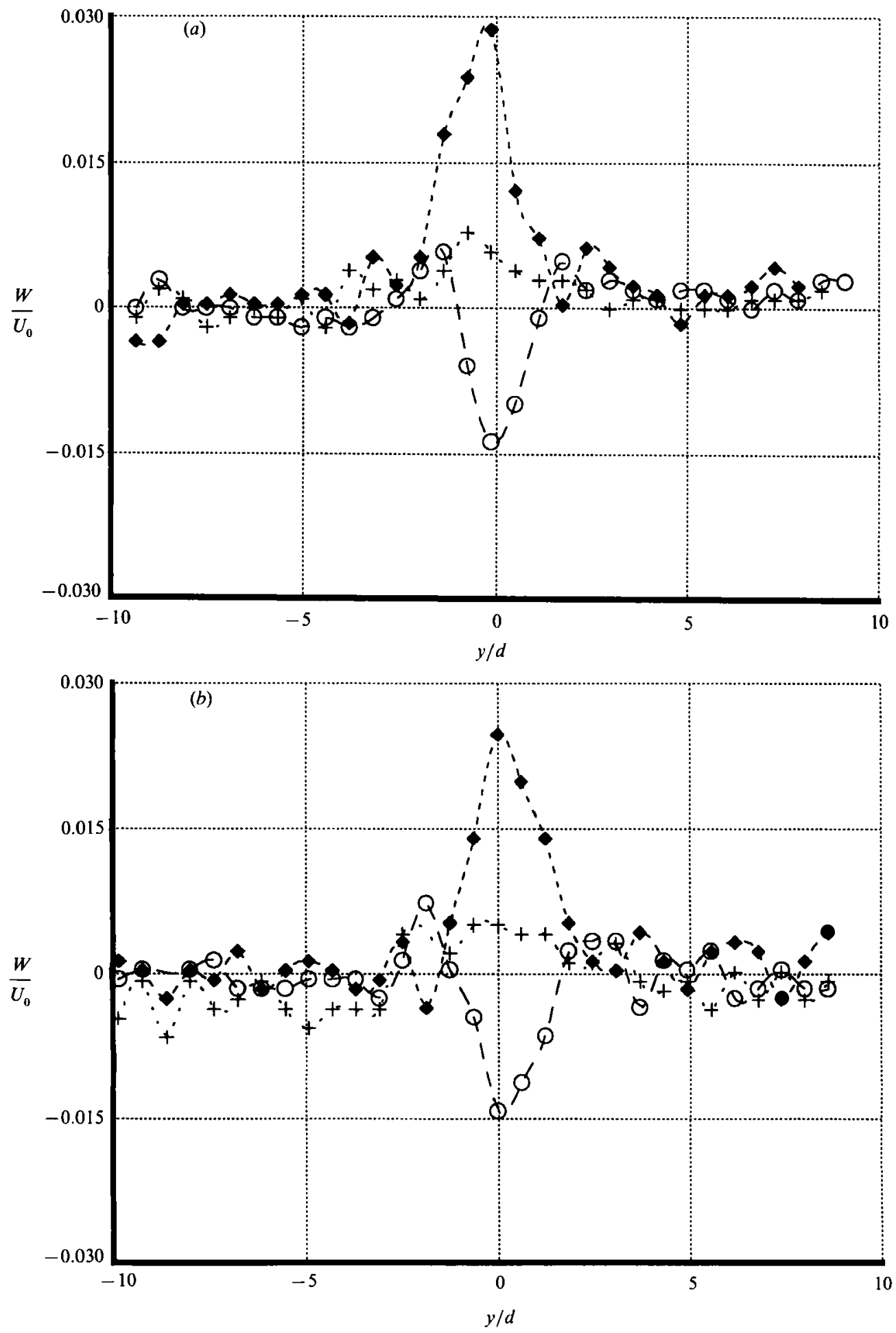

Figure $14(a, b)$. For caption see facing page. 


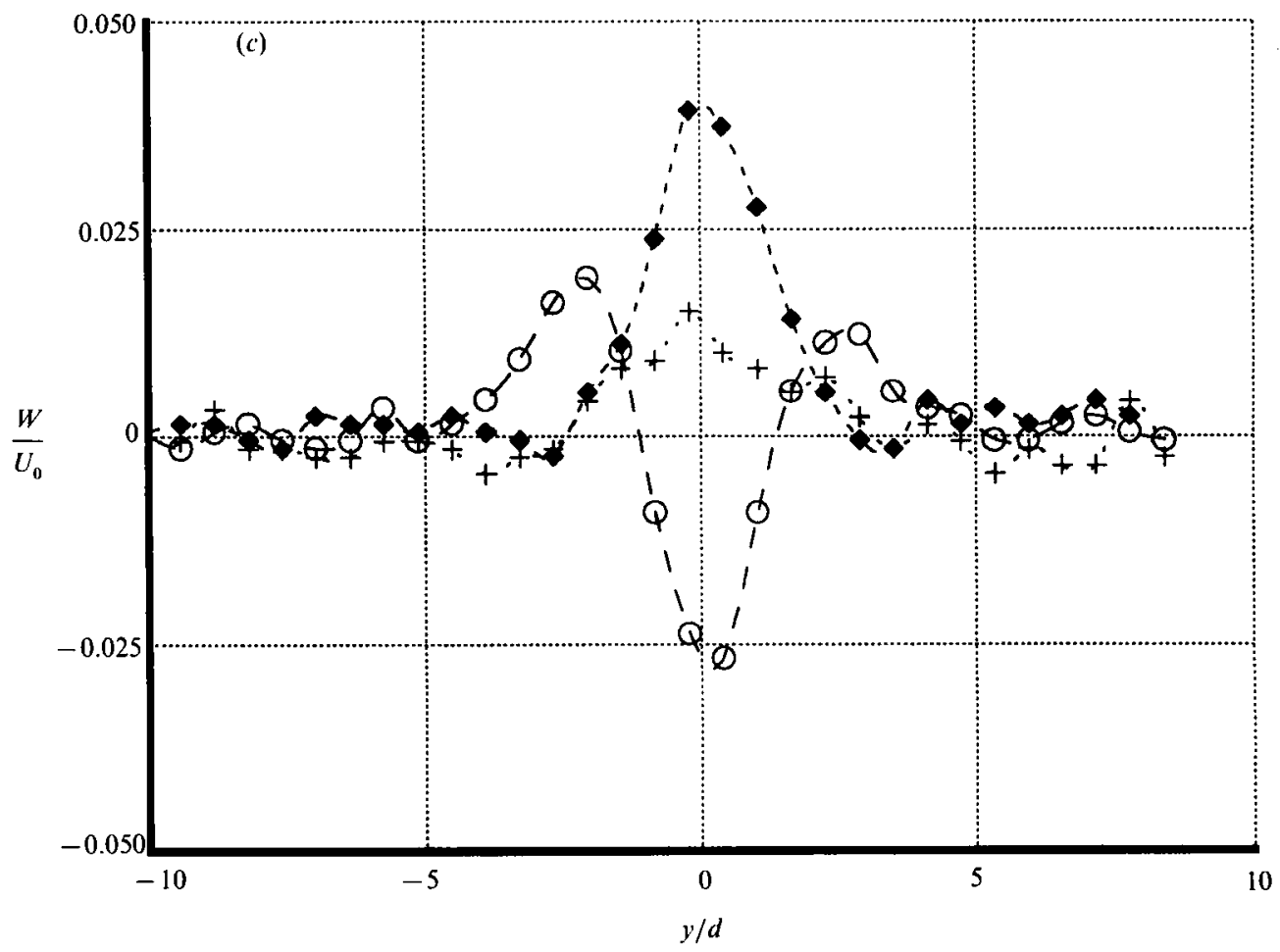

Figure 14. Profiles of the mean spanwise component of velocity at centre-span. $d=0.10 \mathrm{~cm}$ at $R e=100, D=0.47 \mathrm{~cm}, A=190$. (a) $x / d=5,(b) x / d=10,(c) x / d=40$. Symbols same as in figure 12 .

cylinder and found that parallel vortex shedding could not be achieved. No control over the wake of the main cylinder could be achieved in this configuration.

These experiments show that oblique shedding is not due to a secondary instability of the wake, but rather due to a pressure gradient at the back stagnation region of the cylinder. This pressure mismatch could be caused by uneven formation of horseshoe vortices in the wall boundary layers upstream of the cylinder. The wrapping of these vortices around the cylinder can greatly alter the pressure field in the base region. These horseshoc vortices interact with the Kármán vortex street in the form of shedding cells as discussed by Gerich \& Eckelmann (1982). This interaction occurs downstream of the base region and one might expect that the crucial part of the flow that causes the oblique shedding is that entrained into the immediate base region due to the pressure mismatch at the two ends of the cylinder. Therefore, the oblique shedding should also be associated with a spanwise flow at the base of the cylinder. The existence and orientation of such a flow is dictated by the sign of the pressure gradient and can be verified only by a direct measurement of the spanwise component of velocity itself. The need to resolve the velocity in the near wake of the cylinder into its streamwise and spanwise components required the implementation of the frequency shifted laser-Doppler velocimetry (LDV). Hotwire anemometry would only resolve the magnitude of the velocity and not its direction. The LDV measurements were conducted in a water tunnel and the results are discussed below. 


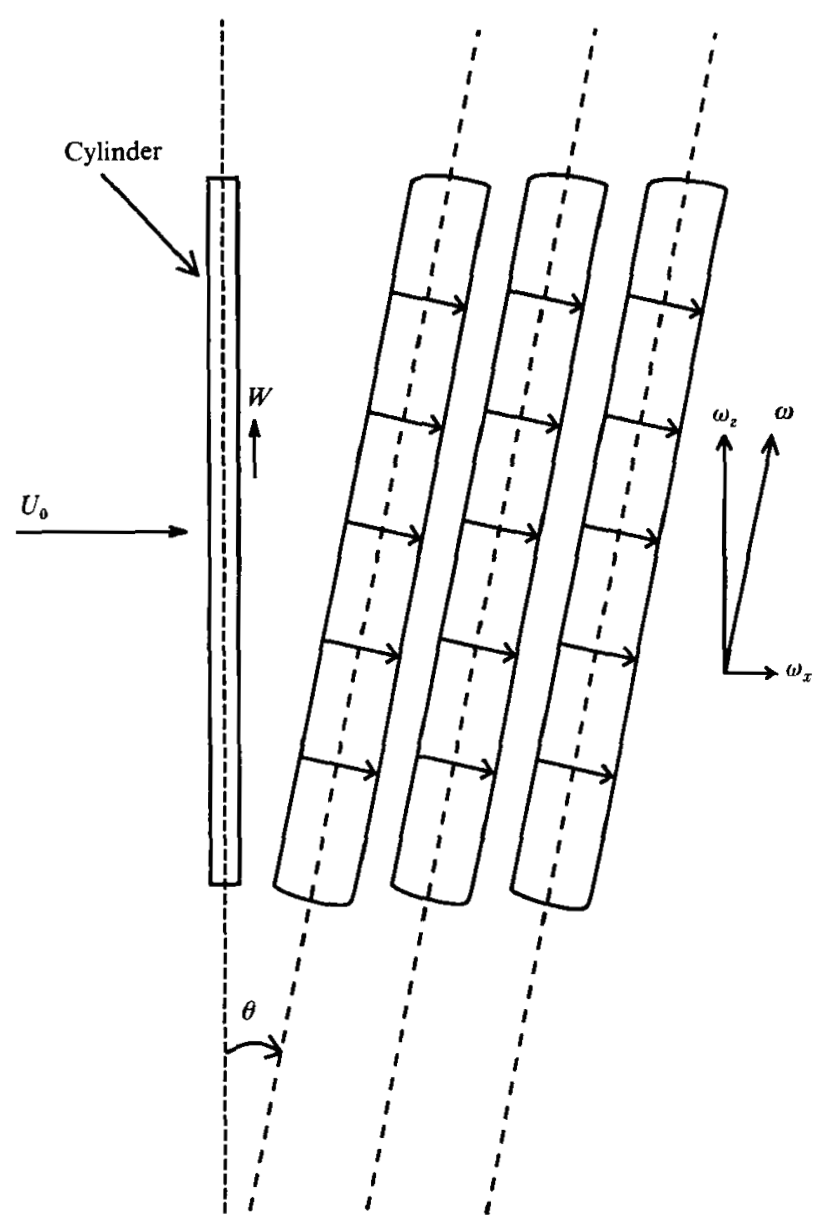

FIGURe 15. Sketch of the mean base velocity for oblique vortex shedding, $\theta<0$, and corresponding vorticity vector on the top side of the vortex street.

\subsection{Measurements of the spanwise velocity component in parallel and oblique vortex streets}

A frequency-shifted two-component fibre-optics LDV system was used to measure velocity profiles across the wake at various streamwise and spanwise stations. The mean spanwise component of velocity, $W$, was measured in the wake of the main cylinder for different angles of shedding. Figure 12 shows profiles taken across the wake at $z=0, x / d=1$, which is inside the closure region of the near wake, at $R e=100$. As predicted, the oblique shedding shows a finite spanwise mean velocity component, whereas the parallel shedding shows no base flow. The orientation of the $W$-velocity is in agreement with the sign of the pressure gradient, i.e. $W>0$ for $\theta<0$ and $W<0$ for $\theta>0$.

More evidence of the existence of a spanwise flow in the cylinder base region is obtained from velocity measurements of curved vortex shedding. By using the control cylinders such that $L_{1}=L_{2}=0$, we induced the case of curved vortex shedding depicted in figure $5(a)$. We chose this condition of vortex shedding because the angle of the vortices can be considered positive for $z<0$, negative for $z>0$ and 


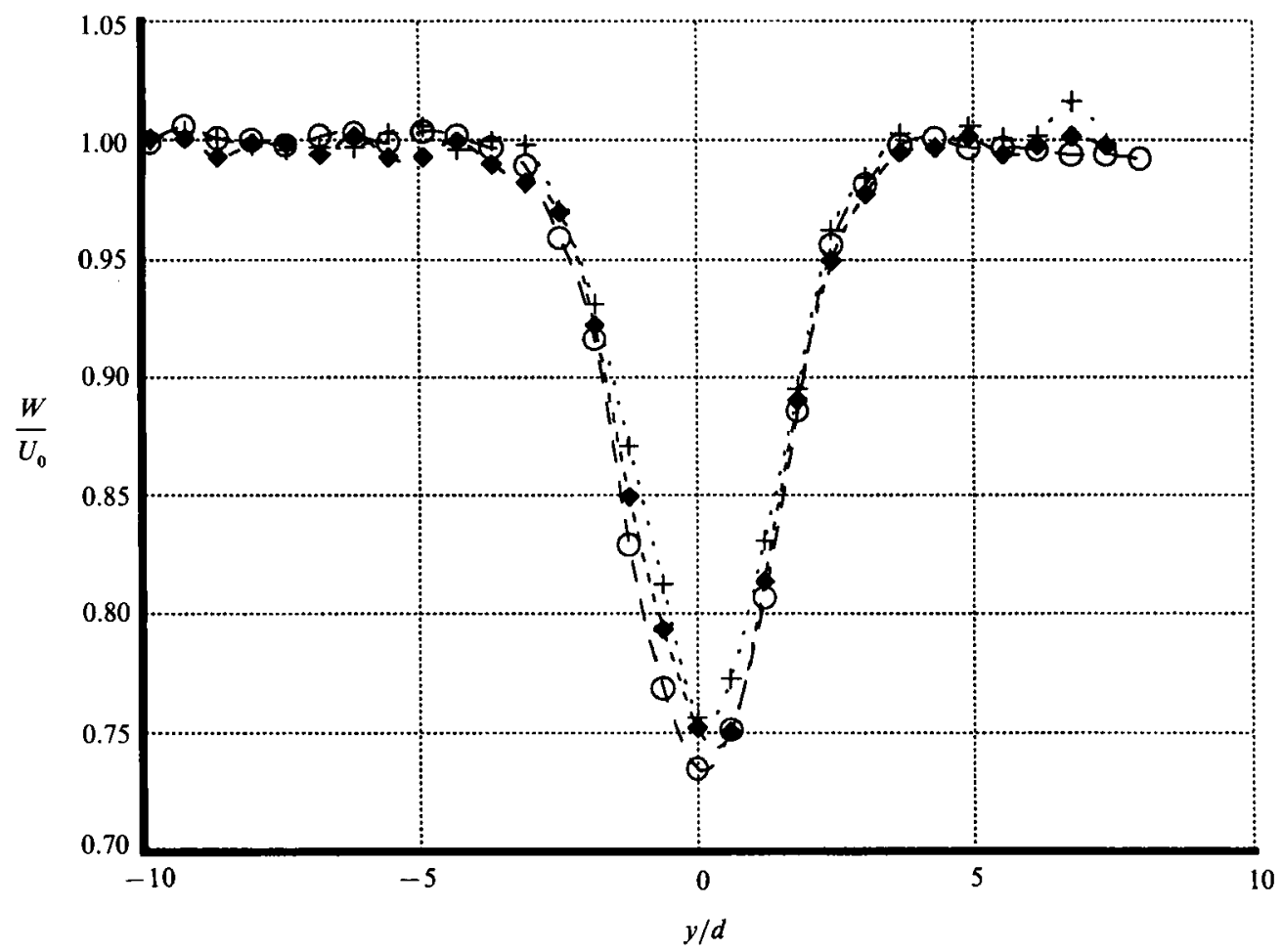

Figure 16. Profiles of the mean $U$-component of velocity across the wake. $d=0.10 \mathrm{~cm}, R e=100$, $D=0.47 \mathrm{~cm}, A=190, x / d=40$. The corresponding $W$-component profile is in figure $14(c)$. Symbols same as in figure 12 .

zero at $z=0$. The base flow should therefore be directed from the centre of the span toward the cylinder ends. $W$-velocity profiles were measured across the wake for this case of vortex shedding at several stations along the span and at $x / d=10, R e=100$. Three of these profiles, namely at $z / d=0,-45$ and 45 , are presented in figure 13 . The profiles clearly indicate that there is a finite mean spanwise flow on both sides of the mid-span, with $W>0$ along the upper half of the cylinder and $W<0$ along the lower half, in agreement with the sign of the local angle of vortex shedding. The middle of the span itself shows no spanwise velocity, since the vortex filaments are locally parallel to the cylinder axis at that location.

The evolution of the $W$-velocity profiles for the oblique and parallel shedding at $x / d=5,10$ and 40 are presented in figure 14. The main feature to observe as the profiles evolve downstream is the formation of opposite-sign shoulders around $|y / d| \approx 2.5$. These shoulders are most prominent for the case of $\theta>0$ at $x / d=40$, where they indicate that a mean reversed spanwise flow is induced in the outer edges of the wake due to the tilting effect.

While spanwise velocity in the developed region of the wake can be attributed to the induced velocity of the oblique vortices, its existence in the base region, where vortices are not yet formed, is attributed to a pressure difference between the ends of the cylinder. The spanwise flow in the base region of the cylinder generates a crossshear near the sensitive separation region, thereby affecting the angle of the shed vortices.

In the case of oblique shedding, the $W$-velocity gradient generates a streamwise 


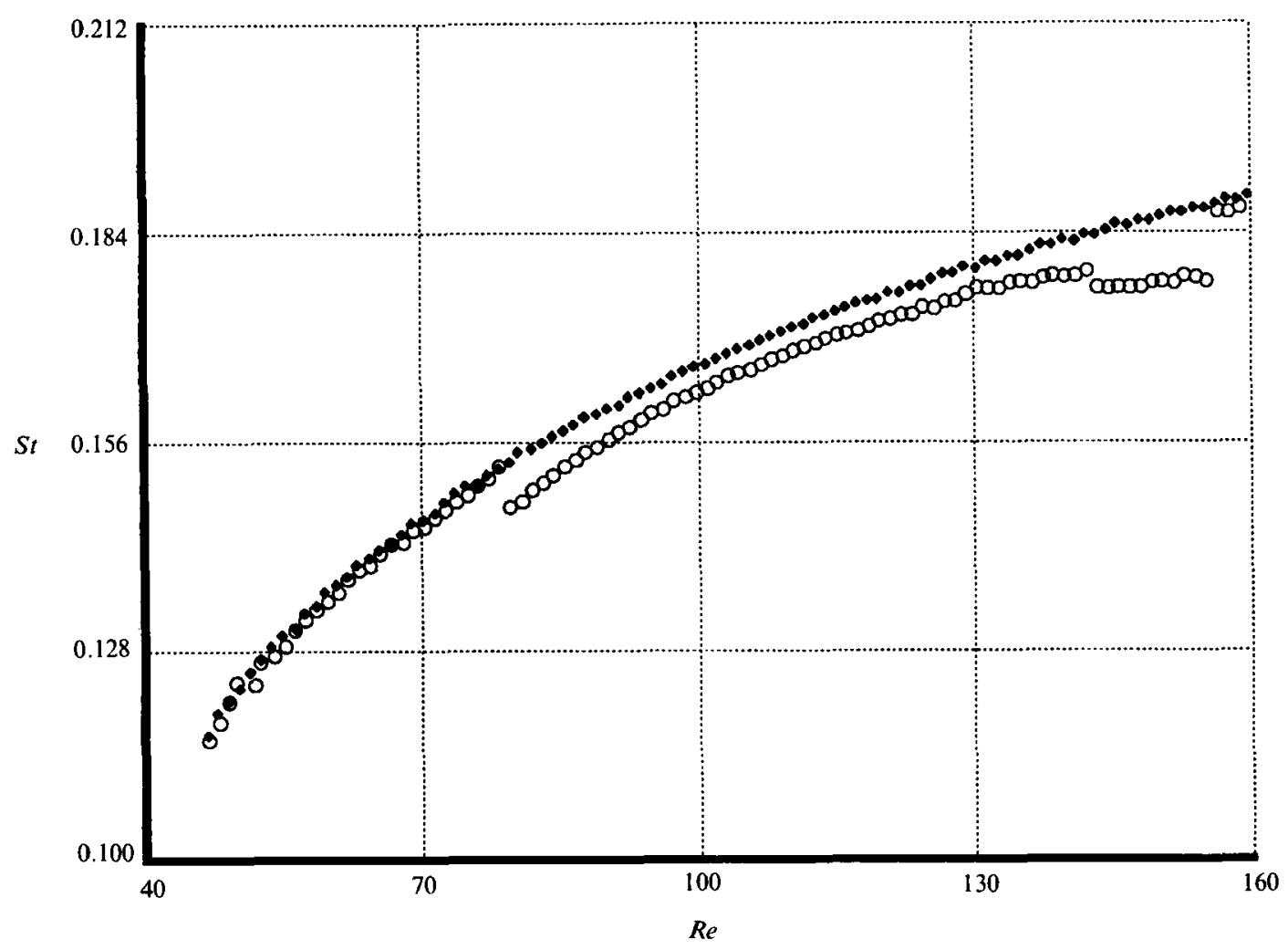

FigURE 17. St-Re data for a cylinder of diameter $d=0.08 \mathrm{~cm}$. $O$, without control cylinders, aspect ratio $=750 ; \diamond$, parallel shedding obtained with control cylinders, $D=0.63 \mathrm{~cm}, A=265$.

vorticity vector $\omega_{x}$ in the wake, which is a component of the total vorticity vector $\omega$. The angle between $\omega$ and the $z$-component, $\omega_{z}$, determines the angle of shedding $\theta$. If one considers the case of oblique shedding with $\theta<0$, the vortices peel off from the top end of the cylinder first, then the mean base flow is directed upward at the centre of the wake and downward at the outer edges of the wake, in agreement with the pressure and base flow data presented earlier. This is illustrated in figure 15 . The quantity $\partial W / \partial y$ generates a streamwise cross-shear which yields the correct resulting vorticity when added to the spanwise vorticity component. The angle of vortex shedding is then determined by the angle formed by $\omega$ with respect to the cylinder axis. In the case of parallel shedding, the vorticity vector $\omega$ in the wake does not have a streamwise component because there is no $W$-velocity.

Note from figure 15 that:

$$
\tan (\theta)=\omega_{x} / \omega_{z}
$$

We could then substitute for $\omega_{x}$ and $\omega_{z}$ the approximate quantities $S_{W}=\partial W /\left.\partial y\right|_{\max }$ and $S_{U}=\partial U /\left.\partial y\right|_{\max }$, respectively, which are the maximum slopes of the $W$-and $U$ velocity profiles, measured at the inflexion points. The angle of shedding could then be calculated from the expression

$$
\theta \approx \tan ^{-1}\left(S_{w} / S_{U}\right)
$$

With this simple method, the angle $\theta$ was calculated from the velocity profiles at $x / d=40$. The $W$-component profile is shown in figure $14(c)$ and the $U$-component 
profile is in figure 16. The angle obtained from this simple calculation was found to be within $2^{\circ}$ of the measured value.

\subsection{The Strouhal-Reynolds number relationship for parallel and oblique vortex shedding}

As has been previously stated, the oblique vortex shedding is accompanied by discontinuities in the $S t-R e$ curve. However, it was observed by Williamson (1988) that inducing parallel shedding results in a continuous $S t-R e$ curve. This result was also obtained when the control cylinders were used by Hammache \& Gharib (1989) to control the vortex shedding, as shown in figure 17. The parallel-shedding data points are best fitted by the following relation:

$$
S t_{0}=0.212-5.35 / R e .
$$

As far as parallel shedding is concerned, the above relation is in relatively good agreement with the results of Williamson (1988) and Konig, Eisenlohr \& Eckelmann (1990).

\section{Conclusions}

We have developed a control technique which permitted us to create vortex shedding of different geometries and study the mechanisms that generate oblique shedding. This technique proved to be much easier to use than other currently available techniques and showed no need for iteration as the free-stream velocity or cylinder aspect ratio were altered.

The findings in this paper contradict previous interpretations that the oblique shedding is due to a secondary instability of the flow and that its onset is Reynoldsnumber dependent. The pressure data, taken at the base of the cylinder along the span indicated the necessity of a symmetric base pressure distribution for parallel vortex shedding, whereas the oblique shedding is due to a lack of pressure symmetry. The pressure asymmetry in the base region indicates the existence of a spanwise flow in the near wake of the cylinder. Indeed, such a velocity component was measured with the LDV technique for the case of oblique shedding and its sign was found to be in agreement with the sign of the pressure gradient obtained from the base pressure measurements. Parallel shedding indicated no spanwise flow. The pressure measurements alone do not determine the magnitude of the spanwise velocity component, but they do guarantee that a zero pressure gradient is equivalent to zero spanwise flow and, therefore, equivalent to parallel vortex shedding. The magnitude of the spanwise velocity vector, $W$, depends on the boundary and entrainment geometry. For the oblique shedding, the measured gradient of $W$ in the $y$-direction is equivalent to a cross-shear in the $x$-direction, which is responsible for a streamwise vorticity component $\omega_{x}$. This additional vorticity component is generated at the base of the cylinder, again due to the base pressure gradient. The total vorticity $\omega$ is directed along the vortex axis, i.e. at an angle $\theta$ (the angle of shedding) with respect to the $z$-direction. In contrast, the case of parallel vortex shedding shows no spanwise flow in the wake and the vorticity vector at any given point of the vortex street is parallel to the cylinder axis.

In a good quality flow and in the absence of any vibrations, we showed that oblique vortex shedding and accompanying discontinuities in the $S t-R e$ curve are due solely to geometrical and flow conditions at the ends of the cylinder. 
The authors are grateful for the support provided by the Office of Naval Research, Program Contract No. N00014-90J-1314, and by DARPA/URI, Program Contract No. N00014-86K-0758.

\section{REFERENCES}

Eisenlohr, H., \& Eckelmann, H. 1989 Vortex splitting and its consequences in the vortex street wake of cylinders at low Reynolds number. Phys. Fluids A 1, 189.

Gaster, M. 1969 Vortex shedding from slender cones at low Reynolds numbers. J. Fluid Mech. $38,565$.

Gerich, D. \& Eckelmanv, H. 1982 Influence of end plates and free ends on the shedding frequency of circular cylinders. J. Fluid Mech. 122, 109.

Hammache, M. \& Giarib, M. 1989 A novel method to promote parallel vortex shedding in the wake of circular cylinders. Phys. Fluids A 1, 1611.

Konia, M., Eisenlohr, H. \& Eckelmann, H. 1990 The fine structure in the Strouhal-Reynolds number relationship of the laminar wake of a circular cylinder. Phys. Fluids. A 2, 1607.

Maull, D. J. \& Young, R. A. 1973 Vortex shedding from bluff bodies in a shear flow. J. Fluid Mech. 60, 401.

Ramberg, S. E. 1983 The effects of yaw and finite length upon the vortex wakes of stationary and vibrating circular cylinders. J. Fluid Mech. 128, 81.

Roshкo, A. 1954 On the development of turbulent wakes from vortex streets. NACA Rep. 1191.

Slaouti, A. \& Gerrard, J. H. 1981 An experimental investigation on the end effects on the wake of a circular cylinder towed through water at low Reynolds numbers. J. Fluid Mech. 112, 297.

Stansby, P. K. 1974 The effect of end plates on the base pressure coefficient of a circular cylinder. Aero. J. 78, 36 .

Tritton, D. J. 1959 Experiments on the flow past a circular cylinder at low Reynolds numbers. J. Fluid Mech. 6, 547.

VAN ATta, C. W. \& GhaRIB, M. 1987 Ordered and chaotic vortex streets behind circular cylinders at low Reynolds numbers. J. Fluid Mech. 174, 113.

Williamson, C. H. K. 1988 Defining a universal and continuous Strouhal-Reynolds number relationship for the laminar vortex shedding of a circular cylinder. Phys. Fluids 31, 2742.

Williamson, C. H. K. 1989 Oblique and parallel modes of vortex shedding in the wake of a circular cylinder at low Reynolds numbers. J. Fluid Mech. 206, 579.

Williamson, C. H. K. \& Roshro, A. 1989 Measurements of base pressure in the wake of a cylinder at low Reynolds numbers. Z. Flugwiss. Weltraumforsch. (submitted). 